\title{
Evaluation of Galerkin Singular Integrals for Anisotropic Elasticity: Displacement Equation
}

\author{
L. J. Gray \\ Computer Science and Mathematics Division \\ Oak Ridge National Laboratory \\ Oak Ridge, TN 37831-6367 \\ A. Griffith \\ Department of Computational and Applied Mathematics \\ Rice University \\ Houston TX 77005 \\ L. Johnson \\ Department of Mathematics \\ Presbyterian University \\ Clinton, SC 29325 \\ P. A. Wawrzynek \\ Fracture Analysis Consultants \\ 121 Eastern Heights Drive \\ Ithaca NY 14850
}

\begin{abstract}
Algorithms for the direct evaluation of singular Galerkin boundary integrals for three-dimensional anisotropic elasticity are presented. The integral of the traction kernel is defined as a boundary limit, and (partial) analytic evaluation is employed to compute the limit. The spherical angle components of the Green's function and its derivatives are not known in closed form, and thus the analytic integration requires a splitting of the kernel, into 'singular' and 'non-singular' terms. For the coincident singular integral, a single analytic evaluation suffices to isolate the potentially divergent term, and to show that this term self-cancels. The implementation for a linear element is considered in detail, and the extension to higher order curved interpolation is also discussed. Results from test calculations establish that the algorithms are successful.
\end{abstract}

\section{Introduction}

A boundary integral formulation for three dimensional anisotropic elasticity was first considered in the 1973 paper by Vogel and Rizzo [39], and this is therefore an appropriate topic for the present volume honoring Professor Rizzo.

Subsequent to the Vogel and Rizzo paper, Wilson and Cruse [41] used interpolation 
from pre-computed Green's function tables to obtain a computationally efficient algorithm. (The book by Schclar [32] contains a survey of work in this area up to 1994). The reason for the computed tables is that, unlike other equations in computational mechanics, the Green's function $\mathcal{U}(P, Q)$ is not known in closed form. Specifically,

$$
\mathcal{U}(P, Q)=\frac{1}{8 \pi^{2} r} \tilde{\mathcal{U}}(P, Q)=\frac{1}{8 \pi^{2} r} \tilde{\mathcal{U}}(\mathbf{R} / r)=\frac{1}{8 \pi^{2} r} \tilde{\mathcal{U}}(\zeta, \varphi),
$$

where $r=\|\mathbf{R}\|=\|Q-P\|$ is the distance between the two points $P$ and $Q$, and $\{\zeta, \varphi\}$ are spherical angles (with respect to a chosen coordinate system) defining the unit direction vector $\mathbf{R} / r$ [41]. Perhaps the nicest derivation of this result can be found in Synge [36]. While several forms can be given for the function $\tilde{\mathcal{U}}$ $[2,19,22,40]$, it cannot be expressed in algebraic form or in terms of the well known special functions; see $[25,27,38]$ for the most recent work in this area, and complete discussions of previous work. In the following, $\tilde{\mathcal{U}}$ will be written as a function of $P$ and $Q, \mathbf{R}$, or spherical angles, whichever is the most convenient at the moment.

The focus herein is on the evaluation of singular integrals that arise in a Galerkin approximation of the displacement boundary integral equation, with most attention on the integrals containing the first derivative of the Green's function. This is a necessary first step in addressing the more difficult hypersingular traction equation and its second order derivatives. The lack of a simple form for the Green's function is clearly an impediment for singular integration algorithms [17, 35, 37]. The special aspect of anisotropic elasticity is that while $\tilde{\mathcal{U}}(P, Q)$ is not a function of $r$, it nevertheless contributes an angular variation of the Green's function in the neighborhood of the singular point $r=0$. Thus, unlike the situation for fundamental solutions given in terms of special functions - e.g., the Bessel function $H_{0}^{1}(k r)$ for the two-dimensional Helmholtz equation - the singular integration cannot be based upon asymptotic expansions [26] at $r=0$. The angular variation in $\tilde{\mathcal{U}}$ must be taken into account, and this obviously presents a challenge for methods based upon analytic integration at the singularity.

The two early papers mentioned above employed a collocation approximation. The Galerkin approximation is advantageous for dealing with the hypersingular equation, and applications in anisotropic elasticity have been reported [4, 23]. A general introduction to the Galerkin method can be found in [5], while [21, 34] are two basic references for elasticity. For a Galerkin approximation in three dimensions, a number of singular integration methods have proved successful in handling the hypersingular kernel: transformation of the integral using Stokes' Theorem $[8,9,10,18]$, and in particular for anisotropic elasticity [4]; numerical methods [33] based upon the Duffy transformation [24]; and analytic integration approaches utilizing either Hadamard Finite Part $[1,6,30,31,33]$ or limit definitions $[12,13]$. While the direct limit analysis is convenient, in that it does not require a reformulation of the integral equations, it does require the ability to 
integrate analytically and to manipulate the integral. It is therefore not obvious that the direct limit approach applies to anisotropic elasticity, and the purpose of this paper is to establish this result. The algorithm presented herein incorporates the basic procedures in [12], together with additional steps necessary to cope with the function $\tilde{\mathcal{U}}$.

The discussion in [12] focused on the hypersingular (two derivatives of the Green's function) integral that appears in the boundary traction equation; however, the techniques apply equally well to the less singular integrals appearing in the displacement equation. Herein we consider the integral involving the first derivative of the Green's function, usually called the Cauchy Principal Value (CPV) integral, as this simpler integral for anisotropic elasticity warrants a discussion. However, if the boundary limit procedures were to work solely for the displacement equation, they would be of little value. As noted above, the ultimate goal is to establish that these methods are capable of analyzing the more difficult hypersingular kernel. To take a step in this direction, the coincident CPV integral will be treated using the double integration procedure described in [12], even though in this case a single analytic integration would suffice. In addition, the adjacent edge hypersingular procedures in [12] will be shown to be applicable to the adjacent edge integral for the anisotropic CPV kernel.

The remainder of the paper is organized as follows. The next section provides a brief overview of the boundary integral formulation for anisotropic elasticity. Section 3 details the limit evaluation algorithm for the coincident CPV integral, assuming a linear element. The subsequent section discusses the adjacent edge and vertex integrations, and also presents results from a simple test calculation. Section 5 considers the extension of these results to higher order elements, and the paper concludes with some comments about the extension to the hypersingular traction equation.

\section{Boundary Integral Formulation}

The Navier equations for linear elasticity are

$$
\mathcal{C}_{i j k l}\left(u_{k, l j}+u_{l, k j}\right)=0
$$

where $\mathbf{u}=\left(u_{1}, u_{2}, u_{3}\right)$ is the displacement vector and $\mathcal{C}$ are the elastic constants for the material (as usual, indices after the comma indicate partial derivative, and the summation convention is employed). The corresponding boundary integral statement linking surface displacement $\mathbf{u}(Q)$ and traction $\boldsymbol{\tau}(Q)=\sigma \cdot \mathbf{n}(Q)$ is usually written in the form $[39,41]$

$$
\frac{1}{2} \mathbf{u}(P)+\int_{\Sigma}[\mathcal{T}(P, Q) \mathbf{u}(Q)-\mathcal{U}(P, Q) \boldsymbol{\tau}(Q)] \mathrm{d} Q=0,
$$


where the integral involving the traction kernel $\mathcal{T}$ (defined below) is understood in the Cauchy Principal Value (CPV) sense. However, herein we adopt the boundary limit definition of the singular integrals, as this procedure works equally well for the derivative (hypersingular) equation. With this definition, the boundary integral equation can be written as

$$
\mathcal{F}(P)=\lim _{P_{e} \rightarrow P} \int_{\Sigma}\left[\mathcal{T}\left(P_{e}, Q\right) \mathbf{u}(Q)-\mathcal{U}\left(P_{e}, Q\right) \boldsymbol{\tau}(Q)\right] \mathrm{d} Q=0
$$

where $P_{e}$ are points exterior to the domain $\mathcal{D}$ converging to the point $P \in \Sigma$. Ultimately the two equations are the precisely the same, but the singular integration procedures will obviously be different. An exterior limit is chosen for convenience, as it eliminates the 'free term' $\mathbf{u}(P)$ outside the integral that shows up if an interior limit is employed [20]. To complete the specification of the Green's function given in Eq. (1),

$$
\tilde{\mathcal{U}}(P, Q)=\oint_{S^{1}} \mathbf{K}^{-1}(\boldsymbol{\xi}) d s(\boldsymbol{\xi}),
$$

and the Christoffel matrix $\mathbf{K}(\boldsymbol{\xi}), \boldsymbol{\xi} \in \mathcal{R}^{3}$, is defined by $K_{i j}=\mathcal{C}_{i l j m} \xi_{l} \xi_{m}$ [41]. The line integral contour $S^{1}$ is the unit circle in the plane having normal $\mathbf{R} / r$,

$$
S^{1}=S^{1}(P, Q)=\left\{\boldsymbol{\xi} \in \mathcal{R}^{3} \quad \mid\|\boldsymbol{\xi}\|=1, \boldsymbol{\xi} \cdot \mathbf{R}=0\right\} .
$$

Thus, $\tilde{\mathcal{U}}$ depends only on the unit vector $\mathbf{R} / r$, and as noted in Eq. (1), it can be conveniently represented (and computed) as a function of the direction angles $\{\zeta, \varphi\}$ referenced to any choice of spherical coordinate system $(0<\varphi<\pi$ will denote the polar or azimuthal angle, $0<\zeta<2 \pi$ ). In what follows, we will employ spherical coordinates for which a cartesian axis, $X, Y$, or $Z$, is the polar axis, and the flexibility to select among these three coordinate systems will be important.

The Green's function is only weakly singular (integrable), and thus the integral

$$
\lim _{P_{e} \rightarrow P} \int_{\Sigma} \mathcal{U}\left(P_{e}, Q\right) \boldsymbol{\tau}(Q) \mathrm{d} Q=\int_{\Sigma} \mathcal{U}(P, Q) \boldsymbol{\tau}(Q) \mathrm{d} Q
$$

exists for $P$ on the boundary. It therefore suffices to examine the CPV traction kernel $\mathcal{T}(P, Q)$ integral. The procedures described below will work equally well for the $\mathcal{U}$ integral, with obvious simplifications due to the absence of the limit process.

The traction kernel $\mathcal{T}(P, Q)$ in Eq. (4) is obtained from $\mathcal{U}$ in the same manner as the traction $\tau$ is obtained from displacement. Thus,

$$
\mathcal{T}_{i m}=\frac{1}{2} \sum_{j, k, l} \mathcal{C}_{i j k l}\left(\mathcal{U}_{k m, l}+\mathcal{U}_{l m, k}\right) n_{j}
$$

where $\mathcal{U}_{, l}$ denotes the derivative with respect to the $l^{\text {th }}$ component of $Q$,

$$
\mathcal{U}_{, l}(P, Q)=\frac{1}{8 \pi^{2}}\left(-\frac{R_{l}}{r^{3}} \tilde{\mathcal{U}}(P, Q)+\frac{1}{r} \frac{\partial}{\partial q_{l}} \tilde{\mathcal{U}}(P, Q)\right)
$$




$$
=\frac{1}{8 \pi^{2}}\left(-\frac{R_{l}}{r^{3}} \tilde{\mathcal{U}}+\frac{1}{r}\left\{\frac{\partial \tilde{\mathcal{U}}}{\partial \zeta} \frac{\partial \zeta}{\partial q_{l}}+\frac{\partial \tilde{\mathcal{U}}}{\partial \psi} \frac{\partial \psi}{\partial q_{l}}\right\}\right) .
$$

(To simplify notation, the component indices $\{i, j\}$ for the Green's function will be dropped). As $\mathcal{T}$ is a linear combination of $\mathcal{U}_{, l}(P, Q)$, it will suffice to examine the integration of these individual derivatives.

The function $\tilde{\mathcal{U}}(\zeta, \varphi)$ and its partial derivatives are well behaved functions, and can be computed in a number of ways $[2,7,15,41]$. The test calculations presented below will employ the method based upon residue calculation [29].

\subsection{Galerkin Approximation}

A linear element Galerkin approximation is briefly reviewed here, primarily to define notation. Moreover, the singular integration procedures for higher order elements can be based upon the linear analysis, and thus this element will be considered first. Section 5 will describe the extension to curved interpolation. An equilateral triangle parameter space $\{\eta, \xi\}$, where $-1 \leq \eta \leq 1,0 \leq \xi \leq$ $\sqrt{3}(1-|\eta|)$ will be employed to define the interpolation. These parametric variables will denote the outer $P$ integration, while $\left\{\eta^{*}, \xi^{*}\right\}$ will be used for the inner $Q$ integral. The choice of equilateral parameter space will be convenient for the coincident integration, as will be explained in the next section. The three linear shape functions are

$$
\begin{aligned}
& \psi_{1}(\eta, \xi)=\frac{\sqrt{3}(1-\eta)-\xi}{2 \sqrt{3}} \\
& \psi_{2}(\eta, \xi)=\frac{\sqrt{3}(1+\eta)-\xi}{2 \sqrt{3}} \\
& \psi_{3}(\eta, \xi)=\frac{\xi}{\sqrt{3}} .
\end{aligned}
$$

For an element defined by nodal points $\left\{Q_{j}=\left(q_{1}^{j}, q_{3}^{j}, q_{3}^{j}\right)\right\}$, with nodal displacements $\mathbf{u}\left(Q_{j}\right)$, the interpolation of the boundary surface and boundary displacement are therefore given by

$$
\begin{aligned}
& \Sigma\left(\eta^{*}, \xi^{*}\right)=\sum_{j=1}^{3}\left(q_{1}^{j}, q_{2}^{j}, q_{3}^{j}\right) \psi_{j}\left(\eta^{*}, \xi^{*}\right) \\
& \mathbf{u}\left(\eta^{*}, \xi^{*}\right)=\sum_{j=1}^{3} \mathbf{u}\left(Q_{j}\right) \psi_{j}\left(\eta^{*}, \xi^{*}\right),
\end{aligned}
$$

and similarly for $\tau$. 
In a Galerkin approximation, the shape functions are also employed to define weighting functions to enforce the integral equations. Specifically Eq. (4) becomes

$$
\int_{\Sigma} \hat{\psi}_{k}(P) \mathcal{F}(P) \mathrm{d} P=0
$$

where the weight function $\hat{\psi}_{k}(P)$ consists of all shape functions $\psi_{l}(P)$ that are nonzero at a particular node $P_{k}$. The weight function $\hat{\psi}_{k}(P)$ therefore has limited support, being non-zero only on the elements containing $P_{k}$.

For an element $E_{P}$ for the outer $P$ integration, singular integrals (i.e., when $Q=$ $P$ ) occur if the $Q$-element is either coincident with $E_{P}$, or shares a common edge or vertex with $E_{P}$. The coincident singular integration is considered first.

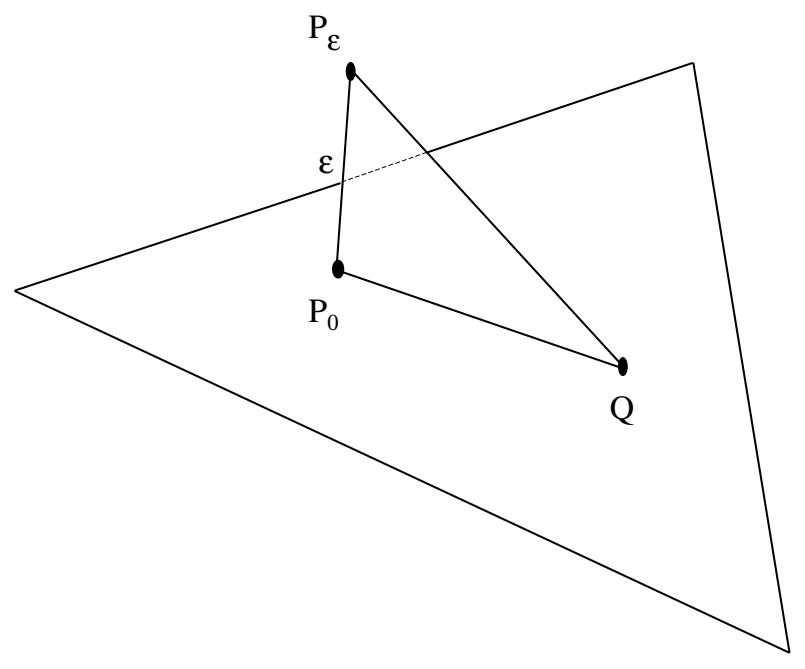

Figure 1: The three points $Q, P_{0}$, and $P_{\epsilon}=P_{0}+\epsilon \mathbf{N}$ that enter into the boundary limit analysis.

\section{Coincident Integration}

From the above discussion, the coincident integral to be evaluated is

$$
\frac{1}{8 \pi^{2}} \int_{E} \hat{\psi}_{k}(P) \int_{E} \mathbf{u}(Q)\left(-\frac{R_{l}}{r^{3}} \tilde{\mathcal{U}}\left(P_{e}, Q\right)+\frac{1}{r} \frac{\partial}{\partial q_{l}} \tilde{\mathcal{U}}\left(P_{e}, Q\right)\right) \mathrm{d} Q \mathrm{~d} P
$$


where the integrals are over an element $E$ defined by the vertices $\left(q_{1}^{m}, q_{2}^{m}, q_{3}^{m}\right)$, $1 \leq m \leq 3$. Formulated as a parameter space integral this becomes

$$
\begin{aligned}
& \frac{J_{P}^{2}}{8 \pi^{2}} \int_{-1}^{1} \int_{0}^{e(\eta)} \psi_{k}(\eta, \xi) \mathrm{d} \eta \mathrm{d} \xi \\
& \quad \int_{-1}^{1} \int_{0}^{e\left(\eta^{*}\right)} \psi_{j}\left(\eta^{*}, \xi^{*}\right)\left(-\frac{R_{l}}{r^{3}} \tilde{\mathcal{U}}\left(P_{\epsilon}, Q\right)+\frac{1}{r} \frac{\partial}{\partial q_{l}} \tilde{\mathcal{U}}\left(P_{\epsilon}, Q\right)\right) \mathrm{d} \eta^{*} \mathrm{~d} \xi^{*},
\end{aligned}
$$

where $e(\eta)=\sqrt{3}(1-|\eta|)$ and $J_{P}$ the (constant) Jacobian. To implement the boundary limit, $P_{e}$ has been replaced by $P_{\epsilon}=P_{0}+\epsilon \mathbf{N}$, where $\mathbf{N}$ is the unit outward normal to the element. This geometry is illustrated in Figure 1, and the subscript 0 has been added to $P$ to emphasize that this point corresponds to $\epsilon=0$.

As the singularity is at $\eta^{*}=\eta, \xi^{*}=\xi$, it makes sense to introduce polar coordinates, replacing $\left\{\eta^{*}, \xi^{*}\right\}$ with $\{\rho, \theta\}$,

$$
\begin{aligned}
& \eta^{*}-\eta=\rho \cos (\theta) \\
& \xi^{*}-\xi=\rho \sin (\theta)
\end{aligned}
$$

as illustrated in Fig. 2. (It would be more appropriate to denote the polar coordinates by $\left(\rho^{*}, \theta^{*}\right)$, but the superscript is dropped to simplify notation). Moreover, as the polar coordinate parameters are physically a radial and an angle coordinate, it is well suited to address the anisotropic Green's function.

With this transformation,

$$
\begin{aligned}
& \mathbf{R}=\left(R_{1}, R_{2}, R_{3}\right)=\left(a_{1} \rho-\epsilon N_{1}, a_{2} \rho-\epsilon N_{2}, a_{3} \rho-\epsilon N_{3}\right) \\
& r^{2}=a^{2} \rho^{2}+\epsilon^{2}
\end{aligned}
$$

where $\mathbf{a}=\left(a_{1}, a_{2}, a_{3}\right)$ is a function of $\theta$,

$$
a_{m}=a_{m}^{c} \cos (\theta)+a_{m}^{s} \sin (\theta),
$$

and $a^{2}=\|\mathbf{a}\|^{2}$. The coefficients $a_{m}^{\alpha}, \alpha=c, s$ depend solely on the coordinates of the element nodes.

As shown in Fig. 2, having a formula for the upper limit of $\rho, \rho_{L}(\theta), 0<\rho<\rho_{L}$, necessitates decomposing the $Q$ parameter space into three subtriangles; however, as discussed in [12], it suffices to consider the lower subtriangle defined by the edge $\xi^{*}=0$. For this subtriangle, the integration limits are $\Theta_{1} \leq \theta \leq \Theta_{2}$ and

$$
\begin{aligned}
\rho_{L} & =-\frac{\xi}{\sin (\theta)} \\
\Theta_{1} & =-\frac{\pi}{2}-\tan ^{-1}\left(\frac{1+\eta}{\xi}\right) \\
\Theta_{2} & =-\frac{\pi}{2}+\tan ^{-1}\left(\frac{1-\eta}{\xi}\right)
\end{aligned}
$$

It is convenient to consider the two constitutents of $\mathcal{T}(P, Q)$, Eq. (14), separately. 


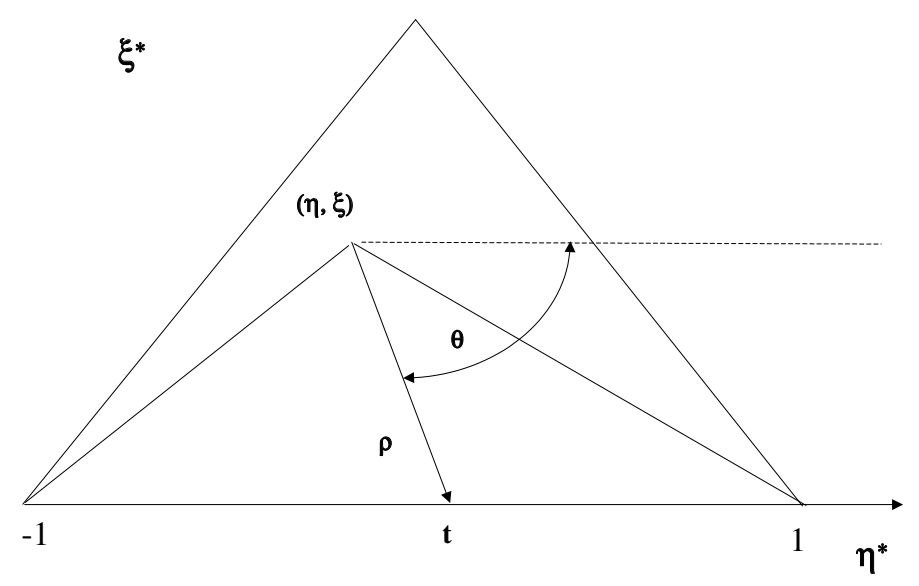

Figure 2: First polar coordinate transformation, $\left\{\eta^{*}, \xi^{*}\right\} \rightarrow\{\rho, \theta\}$, for the coincident integration. The variable $t$ eventually replaces $\theta$.

\section{$3.1 \quad \mathcal{T}$ Kernel: $\tilde{\mathcal{U}}$ term}

With the polar coordinate transformation, the $Q$-shape function is

$$
\psi_{j}\left(\eta^{*}, \xi^{*}\right)=\psi_{j}(\eta, \xi)+c_{j}(\theta, \eta, \xi) \rho,
$$

and the constant term integral

$$
\begin{aligned}
-\frac{J_{P}^{2}}{8 \pi^{2}} \lim _{\epsilon \rightarrow 0} \int_{-1}^{1} \int_{0}^{e(\eta)} \psi_{k}(\eta, \xi) \psi_{j}(\eta, \xi) \mathrm{d} \xi \mathrm{d} \eta \times & \\
& \int_{\Theta_{1}}^{\Theta_{2}} \int_{0}^{\rho_{L}} \rho \frac{a_{l} \rho-\epsilon N_{l}}{\left(a^{2} \rho^{2}+\epsilon^{2}\right)^{3 / 2}} \tilde{\mathcal{U}}\left(P_{\epsilon}, Q\right) \mathrm{d} \rho \mathrm{d} \theta
\end{aligned}
$$

will be examined first. For the $c_{j}(\theta, \eta, \xi) \rho$ term, the integral is finite at $\epsilon=0$, and thus the analysis is much easier; this integral will be discussed at the end of this section.

The goal in Eq. (20) is to integrate $\rho$ analytically and then let $\epsilon \rightarrow 0$, and the obvious roadblock is that an analytic form for $\tilde{\mathcal{U}}\left(P_{\epsilon}, Q\right)$ is unavailable. The first step in getting around this difficulty is to rewrite this function as (see Fig. 1)

$$
\tilde{\mathcal{U}}\left(P_{\epsilon}, Q\right)=\tilde{\mathcal{U}}\left(P_{0}, Q\right)+\left[\tilde{\mathcal{U}}\left(P_{\epsilon}, Q\right)-\tilde{\mathcal{U}}\left(P_{0}, Q\right)\right]
$$


and to note that for the linear element $\left(Q-P_{0}\right) /\left\|Q-P_{0}\right\|=\mathbf{a} / a$. Thus, $\tilde{\mathcal{U}}\left(P_{0}, Q\right)$ is independent of $\rho$, and executing the $\rho$ integration for the first term on the right hand side is straightforward,

$$
\begin{aligned}
& \tilde{\mathcal{U}}\left(P_{0}, Q\right) \int_{0}^{\rho_{L}} \rho \frac{a_{l} \rho-\epsilon N_{l}}{\left(a^{2} \rho^{2}+\epsilon^{2}\right)^{3 / 2}} \mathrm{~d} \rho= \\
& \tilde{\mathcal{U}}\left(P_{0}, Q\right)\left(\frac{a_{l}}{a^{3}} \log \left(2 a \rho_{L}\right)-\frac{N_{l}}{a^{2}}-\frac{a_{l}(1+\log (\epsilon))}{a^{3}}\right) .
\end{aligned}
$$

The $\log (\epsilon)$ term appearing in this expression is precisely the term that the Cauchy Principal Value [14] procedure eliminates by removing a symmetric neighborhood of the singular point. In the boundary limit approach, this term simply cancels on its own,

$$
(1+\log (\epsilon)) \int_{0}^{2 \pi} \tilde{\mathcal{U}}\left(P_{0}, Q\right) \frac{a_{l}}{a^{3}} \mathrm{~d} \theta=0,
$$

as $a_{l}(\pi+\theta)=-a_{l}(\theta)$ and $\tilde{\mathcal{U}}(P, Q)=\tilde{\mathcal{U}}(Q, P)$ implies that $\tilde{\mathcal{U}}(\mathbf{a} / a)=\tilde{\mathcal{U}}(-\mathbf{a} / a)$. This term can therefore be removed from Eq. (22), leaving a finite expression for the limit. Note however that this expression contains an (integrable) logarithmic singularity, as $\rho_{L}$ approaches zero for $\xi$ approaching zero. We will return to this point in Section 3.4.

For the second term from Eq. (21),

$$
\lim _{\epsilon \rightarrow 0} \int_{0}^{\rho_{L}} \frac{\rho\left(a_{l} \rho-\epsilon N_{l}\right)}{\left(a^{2} \rho^{2}+\epsilon^{2}\right)^{3 / 2}}\left[\tilde{\mathcal{U}}\left(P_{\epsilon}, Q\right)-\tilde{\mathcal{U}}\left(P_{0}, Q\right)\right] \mathrm{d} \rho
$$

analytic integration is out of the question. However, the main role of the exact integration is, as illustrated above, to isolate and remove (in this case via the $\theta$ integration) the potentially divergent $\log (\epsilon)$ term. Fortunately, the integral in Eq. (24) is well behaved at $\epsilon=0$ : after rewriting in a form that will allow taking the limit, this integral can be evaluated numerically.

The key observation needed to justify this last statement is that $\tilde{\mathcal{U}}\left(P_{\epsilon}, Q\right)$ is, by simple geometry, a function of $\rho / \epsilon$. Thus, making the change of variables $s=\rho / \epsilon$, the above integral becomes

$$
\lim _{\epsilon \rightarrow 0} \int_{0}^{\rho_{L} / \epsilon} \frac{a_{l} s^{2}-N_{l} s}{\left(1+a^{2} s^{2}\right)^{3 / 2}} g_{0}(s, \theta) \mathrm{d} s=\int_{0}^{\infty} \frac{a_{l} s^{2}-N_{l} s}{\left(1+a^{2} s^{2}\right)^{3 / 2}} g_{0}(s, \theta) \mathrm{d} s .
$$

where, with an abuse of notation,

$$
g_{0}(s, \theta)=\tilde{\mathcal{U}}\left(P_{\epsilon}, Q\right)-\tilde{\mathcal{U}}\left(P_{0}, Q\right) \equiv \tilde{\mathcal{U}}(s)-\tilde{\mathcal{U}}(\mathbf{a}) .
$$

To justify taking the limit, i.e., replacing the upper limit by infinity, the existence of the integral on the right has to be established. However, $g_{0}(s, \theta)$ vanishes at infinity, and thus the integrand decays at least as fast as $s^{-2}$. 
For computation, it is more convenient to work with a finite length interval. Introducing the change of variables $s=\tan (q) / a$, Eq. (25) becomes

$$
\frac{1}{a^{3}} \int_{0}^{\pi / 2} \tan (q) \beta_{l}(q) g_{0}(q, \theta) \mathrm{d} q
$$

where

$$
g_{0}(q, \theta)=\tilde{\mathcal{U}}(\boldsymbol{\beta})-\tilde{\mathcal{U}}(\mathbf{a})
$$

and $\boldsymbol{\beta}=\left(\beta_{1}, \beta_{2}, \beta_{3}\right)$ is

$$
\beta_{j}=a_{j} \sin (q)-a N_{j} \cos (q) .
$$

Finally, note that this expression is independent of $\rho_{L}$, i.e., it holds for all three sub-triangles; thus, for this term it is not necessary to do the 3 -triangle subdivision, and the complete $\theta$ integral over $[0,2 \pi]$ can be considered. Moreover, the inner integration $\{\theta, s\}$ is independent of $\{\eta, \xi\}$, and thus this nonsingular term is

$$
-\frac{J_{P}^{2}}{8 \pi^{2}} \alpha_{k j} \int_{0}^{2 \pi} \frac{1}{a^{3}} \mathrm{~d} \theta \int_{0}^{\pi / 2} \tan (q) \boldsymbol{\beta}(q) g_{0}(q, \theta) \mathrm{d} q,
$$

where, setting $e(\eta)=1-|\eta|$,

$$
\begin{aligned}
\alpha_{k j} & =\int_{-1}^{1} \int_{0}^{e(\eta)} \psi_{k}(\eta, \xi) \psi_{j}(\eta, \xi) \mathrm{d} \xi \mathrm{d} \eta \\
& = \begin{cases}\sqrt{3} / 6 & k=j \\
\sqrt{3} / 12 & k \neq j\end{cases}
\end{aligned}
$$

As an example, Figure 3 plots $g_{0}(q, \theta)$ for the diagonal components of the Green's function; the elastic constants are for silicon $\left(C_{11}=1.657, C_{12}=0.639, C_{44}=\right.$ $0.796), \mathbf{N}=(1,0,0)$ and $\mathbf{a}=(0,1,0)$. Clearly, there is no problem in evaluating Eq. (30) numerically.

Recall that thus far only the constant term from the shape function $\psi_{j}\left(\eta^{*}, \xi^{*}\right)$ has been considered. However, for the linear term (and obviously any higher power if a curved interpolation is employed) this integral is well defined at $\epsilon=0$. Thus, setting $\epsilon=0$ and carrying out the $\rho$ integration, Eq. (20) becomes

$$
-\frac{J_{P}^{2}}{8 \pi^{2}} \int_{-1}^{1} \int_{0}^{e(\eta)} \psi_{k}(\eta, \xi) \mathrm{d} \eta \mathrm{d} \xi \int_{\Theta_{1}}^{\Theta_{2}} c_{j}(\theta, \eta, \xi) \rho_{L}(\theta) \frac{a_{l}}{a^{3}} \tilde{\mathcal{U}}(\mathbf{a}) \mathrm{d} \theta
$$

The fact that only the constant component of the shape function contributes a 'singular term', and moreover that this limit term eventually reduces to an innocuous integral, is the expected behavior for this 'CPV' integral.

Recapping, the boundary limit algorithm for anisotropic elasticity first splits the angle dependent part of the kernel function as in Eq. (21). The 'singular' term, obtained by replacing $\tilde{\mathcal{U}}\left(P_{\epsilon}, Q\right)$ by $\tilde{\mathcal{U}}(\mathbf{a})$, contains all of the singularity, and can 


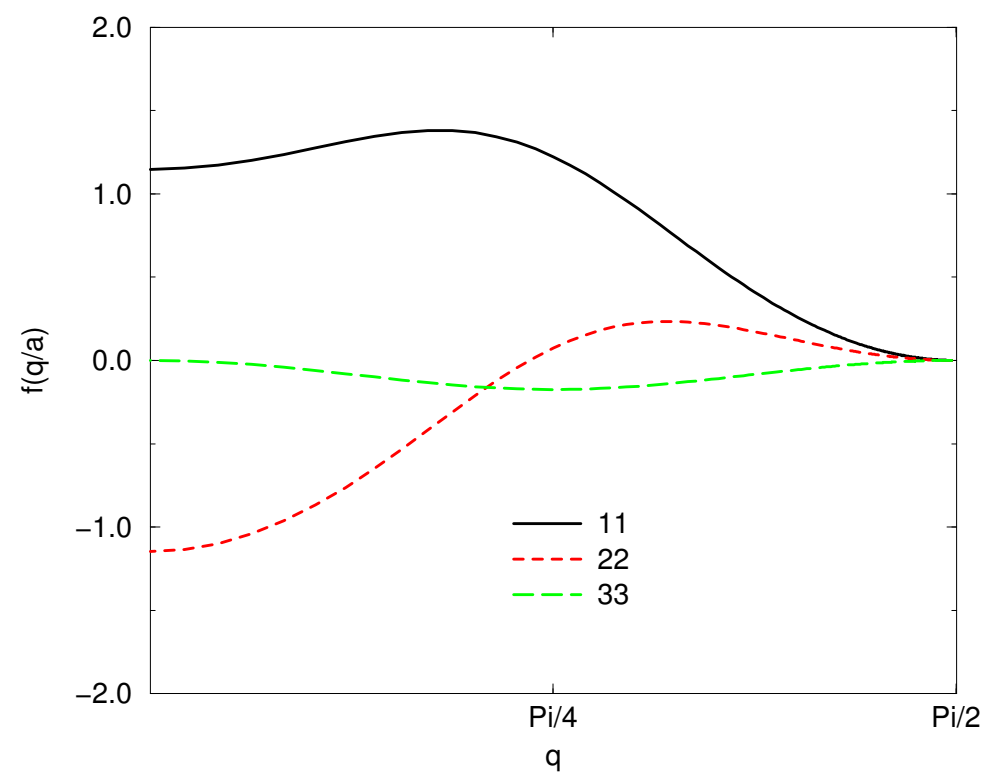

Figure 3: The diagonal components $g_{0}(q)=\tilde{\mathcal{U}}_{k k}\left(P_{\epsilon}, Q\right)-\tilde{\mathcal{U}}_{k k}\left(P_{0}, Q\right)$ for silicon elastic constants.

be treated by the methods in [12]. The remainder term is 'non-singular' and can be expressed as a computable integral. This process will now be applied to the second term in Eq. (14). The $c_{j}(\theta, \eta, \xi) \rho$ term from the $Q$-shape function is, as above, relatively easy, and thus only the constant term will be investigated.

\section{$3.2 \mathcal{T}$ Kernel: derivative of $\tilde{\mathcal{U}}$ term}

Corresponding to Eq. (20), the second integral is

$$
\begin{aligned}
\frac{J_{P}^{2}}{8 \pi^{2}} \lim _{\epsilon \rightarrow 0} & \int_{-1}^{1} \int_{0}^{e(\eta)} \psi_{k}(\eta, \xi) \psi_{j}(\eta, \xi) \mathrm{d} \eta \mathrm{d} \xi \\
& \int_{\Theta_{1}}^{\Theta_{2}} \int_{0}^{\rho_{L}} \frac{\rho}{\left(a^{2} \rho^{2}+\epsilon^{2}\right)^{1 / 2}} \frac{\partial}{\partial q_{l}} \tilde{\mathcal{U}}\left(P_{\epsilon}, Q\right) \mathrm{d} \rho \mathrm{d} \theta
\end{aligned}
$$

and analogously to Eq. (21) the derivative of $\tilde{\mathcal{U}}$ is split as

$$
\frac{\partial}{\partial q_{l}} \tilde{\mathcal{U}}\left(P_{\epsilon}, Q\right)=\frac{\partial}{\partial q_{l}} \tilde{\mathcal{U}}\left(P_{0}, Q\right)+\frac{\partial}{\partial q_{l}}\left[\tilde{\mathcal{U}}\left(P_{\epsilon}, Q\right)-\tilde{\mathcal{U}}\left(P_{0}, Q\right)\right] .
$$


Expanding the first term on the right in terms of angle derivatives, the $\rho$ integration is

$$
\begin{aligned}
\frac{\partial \tilde{\mathcal{U}}}{\partial \zeta}\left(P_{0}, Q\right) & \int_{0}^{\rho_{L}} \frac{\rho}{\left(a^{2} \rho^{2}+\epsilon^{2}\right)^{1 / 2}} \frac{\partial \zeta}{\partial q_{l}}\left(P_{0}, Q\right) \mathrm{d} \rho+ \\
& \frac{\partial \tilde{\mathcal{U}}}{\partial \varphi}\left(P_{0}, Q\right) \int_{0}^{\rho_{L}} \frac{\rho}{\left(a^{2} \rho^{2}+\epsilon^{2}\right)^{1 / 2}} \frac{\partial \varphi}{\partial q_{l}}\left(P_{0}, Q\right) \mathrm{d} \rho .
\end{aligned}
$$

as $\tilde{\mathcal{U}}_{, \zeta}\left(P_{0}, Q\right)$ and $\tilde{\mathcal{U}}_{, \varphi}\left(P_{0}, Q\right)$ are independent of $\rho$. The angle derivatives depend upon the chosen spherical coordinate system (and this choice will be discussed below); a listing of the formulas can be found in the appendix. To take a specific example, namely $l=1$ and $Z$ as the polar axis,

$$
\begin{aligned}
\frac{\partial \zeta}{\partial q_{1}}\left(P_{0}, Q\right) & =-\left.\frac{q_{2}-p_{2}}{\left(q_{1}-p_{1}\right)^{2}+\left(q_{2}-p_{2}\right)^{2}}\right|_{\epsilon=0}=-\frac{1}{\rho} \frac{a_{2}}{a_{1}^{2}+a_{2}^{2}} \\
\frac{\partial \varphi}{\partial q_{1}}\left(P_{0}, Q\right) & =\left.\frac{\left(q_{1}-p_{1}\right)\left(q_{3}-p_{3}\right)}{r_{0}^{2}\left[\left(q_{1}-p_{1}\right)^{2}+\left(q_{2}-p_{2}\right)^{2}\right]^{1 / 2}}\right|_{\epsilon=0}=\frac{1}{\rho} \frac{a_{1} a_{3}}{a^{2} \sqrt{a_{1}^{2}+a_{2}^{2}}}
\end{aligned}
$$

where $r_{0}=\left\|Q-P_{0}\right\|$. All angle derivatives, evaluated at $\left(P_{0}, Q\right)$ are in fact of the form $\gamma / \rho$. The $\rho$ integrand is therefore $1 / \sqrt{a^{2} \rho^{2}+\epsilon^{2}}$, and Eq. (35) is found to be

$$
\left[-\frac{\partial \tilde{\mathcal{U}}}{\partial \zeta}\left(P_{0}, Q\right) \frac{a_{2}}{a_{1}^{2}+a_{2}^{2}}+\frac{\partial \tilde{\mathcal{U}}}{\partial \varphi}\left(P_{0}, Q\right) \frac{a_{1} a_{3}}{a^{2} \sqrt{a_{1}^{2}+a_{2}^{2}}}\right]\left(\frac{\log \left(2 a \rho_{L}\right)}{a}-\frac{\log (\epsilon)}{a}\right) .
$$

The $\log (\epsilon)$ term self-cancels in the integration over $\theta$, as in Eq. (23). This will follow again from the symmetry $\tilde{\mathcal{U}}(-\mathbf{a})=\tilde{\mathcal{U}}(\mathbf{a})$, or equivalently $\tilde{\mathcal{U}}(\zeta, \varphi)=\tilde{\mathcal{U}}(\zeta+$ $\pi, \pi-\varphi)$, as differentiating yields

$$
\begin{aligned}
\frac{\partial \tilde{\mathcal{U}}}{\partial \zeta}(\zeta+\pi, \pi-\varphi) & =\frac{\partial \tilde{\mathcal{U}}}{\partial \zeta}(\zeta, \varphi) \\
\frac{\partial \tilde{\mathcal{U}}}{\partial \varphi}(\zeta+\pi, \pi-\varphi) & =-\frac{\partial \tilde{\mathcal{U}}}{\partial \varphi}(\zeta, \varphi) .
\end{aligned}
$$

The second term in Eq. (34) results in a $\rho$ integral of the form

$$
\int_{0}^{\rho_{L}} \frac{\rho}{\left(a^{2} \rho^{2}+\epsilon^{2}\right)^{1 / 2}} \frac{\partial}{\partial q_{l}}\left[\tilde{\mathcal{U}}\left(P_{\epsilon}, Q\right)-\tilde{\mathcal{U}}\left(P_{0}, Q\right)\right] \mathrm{d} \rho
$$

and from the analysis in the previous section it will come as no surprise that this integral is well behaved at $\epsilon=0$. While it is treated in the same manner as above, the details are somewhat different and, moreover, depend upon the value of $l$ and the spherical coordinate system. For illustrative purposes, we again choose the polar axis for spherical coordinates along $Z, l=1$, and only the $\zeta$ derivative term will be discussed in detail. From Eq. (36),

$$
\frac{\partial \zeta}{\partial q_{1}}\left(P_{\epsilon}, Q\right)=-\frac{a_{2} \rho-\epsilon N_{2}}{\left(a_{1} \rho-\epsilon N_{1}\right)^{2}+\left(a_{2} \rho-\epsilon N_{2}\right)^{2}}
$$


and thus again changing variables from $\rho$ to $s$, the $\zeta$ derivative term from Eq. (39) becomes

$$
\begin{gathered}
\frac{\rho}{\left(a^{2} \rho^{2}+\epsilon^{2}\right)^{1 / 2}}\left(\frac{\partial \tilde{\mathcal{U}}}{\partial \zeta}\left(P_{\epsilon}, Q\right) \frac{\partial \zeta}{\partial q_{l}}\left(P_{\epsilon}, Q\right)-\frac{\partial \tilde{\mathcal{U}}}{\partial \zeta}\left(P_{0}, Q\right) \frac{\partial \zeta}{\partial q_{l}}\left(P_{0}, Q\right)\right) \\
=\frac{1}{\left(1+a^{2} s^{2}\right)^{1 / 2}}\left(\frac{\partial \tilde{\mathcal{U}}}{\partial \zeta}(\mathbf{a}) \frac{a_{2}}{a_{1}^{2}+a_{2}^{2}}-\frac{\partial \tilde{\mathcal{U}}}{\partial \zeta}(s) \frac{s\left(a_{2} s-N_{2}\right)}{D_{s}^{2}}\right)
\end{gathered}
$$

where $D_{s}^{2}=\left(a_{1} s-N_{1}\right)^{2}+\left(a_{2} s-N_{2}\right)^{2}$. Rewriting the term inside the parentheses as

$$
\left[\left(\frac{\partial \tilde{\mathcal{U}}}{\partial \zeta}(\mathbf{a})-\frac{\partial \tilde{\mathcal{U}}}{\partial \zeta}(s)\right) \frac{a_{2}}{a_{1}^{2}+a_{2}^{2}}+\frac{\partial \tilde{\mathcal{U}}}{\partial \zeta}(s)\left(\frac{a_{2}}{a_{1}^{2}+a_{2}^{2}}-\frac{s\left(a_{2} s-N_{2}\right)}{D_{s}^{2}}\right)\right],
$$

it is seen that both terms vanish at least as fast as $s^{-1}$ for $s \rightarrow \infty$. The integration over $\left[0, \rho_{L} / \epsilon\right]$ can therefore be replaced by $0<s<\infty$. Employing the transformation $s(q)=\tan (q) / a$ as above, the $\zeta$-derivative term in Eq. (39) becomes

$$
\frac{1}{a} \int_{0}^{\pi / 2} \sec (q)\left[g_{1, \zeta}^{s}(q) \frac{\partial \tilde{\mathcal{U}}}{\partial \zeta}(\boldsymbol{\beta})+g_{1, \zeta}^{a}\left(\frac{\partial \tilde{\mathcal{U}}}{\partial \zeta}(\boldsymbol{\beta})-\frac{\partial \tilde{\mathcal{U}}}{\partial \zeta}(\mathbf{a})\right)\right] \mathrm{d} q
$$

where $\boldsymbol{\beta}$ is defined in Eq. (29) and

$$
D_{q}^{2}=\beta_{1}^{2}(q)+\beta_{2}^{2}(q) .
$$

The functions $g_{1, \zeta}^{a}$ and $g_{1, \zeta}^{s}$ (for the $Z$ coordinate system) are

$$
\begin{aligned}
g_{1, \zeta}^{a} & =\rho \frac{\partial \zeta}{\partial q_{1}}\left(P_{0}, Q\right)=-\frac{a_{2}}{a_{1}^{2}+a_{2}^{2}} \\
g_{1, \zeta}^{s}(q) & =\sin (q)\left(\left.g_{1, \zeta}^{a}\right|_{a_{j}=\beta_{j}(q)}\right)-g_{1, \zeta}^{a} \\
& =\frac{a_{2}}{a_{1}^{2}+a_{2}^{2}}-\sin (q) \frac{\beta_{2}(q)}{D_{q}^{2}} .
\end{aligned}
$$

Note that for $q \rightarrow \pi / 2, \boldsymbol{\beta} \rightarrow \mathbf{a}$, and thus $g_{1, \zeta}^{s}(q) \rightarrow 0$. Consequently the $\sec (q)$ factor in the above integrals is not a problem. As an example, Fig. 4 plots the integrand of the second term in Eq. (43) as a function of $q$, for the same parameters as in Fig. 3.

The $\varphi$-derivative term takes precisely the same form, and thus from Eq. (36), the corresponding functions $g_{1, \varphi}^{a}$ and $g_{1, \varphi}^{s}$ are

$$
\begin{aligned}
g_{1, \varphi}^{a} & =\rho \frac{\partial \varphi}{\partial q_{1}}\left(P_{0}, Q\right)=\frac{a_{1} a_{3}}{a^{2}\left(a_{1}^{2}+a_{2}^{2}\right)^{1 / 2}} \\
g_{1, \varphi}^{s}(q) & =\sin (q)\left(\left.g_{1, \varphi}^{a}\right|_{a_{j}=\beta_{j}(q)}\right)-g_{1, \varphi}^{a} \\
& =\sin (q) \frac{\beta_{1}(q) \beta_{3}(q)}{a^{2} D_{q}}-\frac{a_{1} a_{3}}{a^{2}\left(a_{1}^{2}+a_{2}^{2}\right)^{1 / 2}} .
\end{aligned}
$$




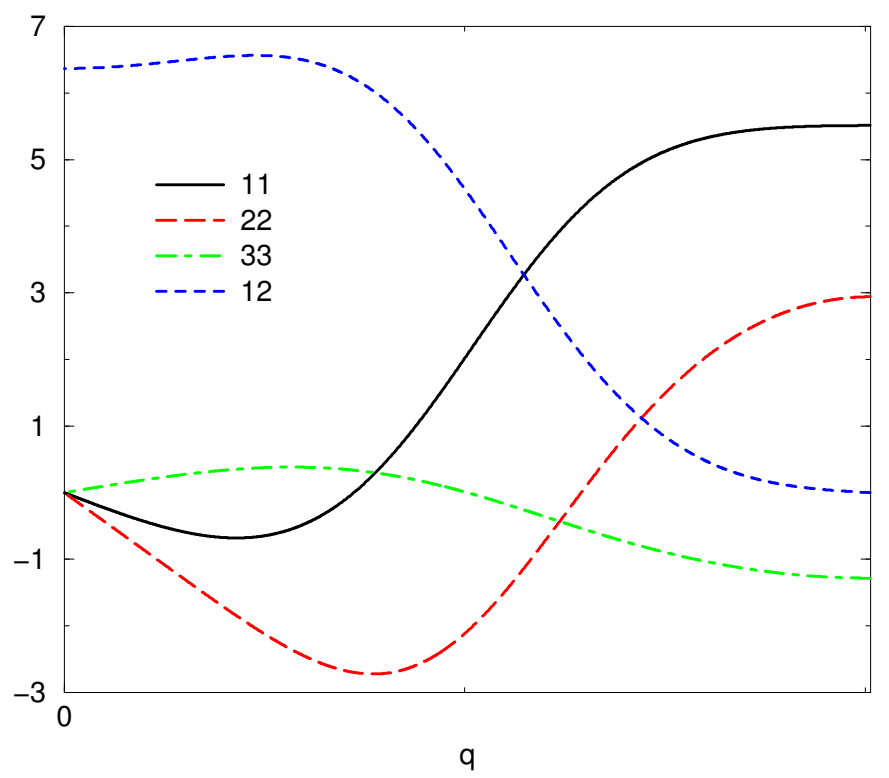

Figure 4: The components of the second term in Eq. (43) as a function of $q$ for silicon elastic constants.

In general, the functions $g_{l, \zeta}$ and $g_{l, \varphi}$ for $l=1,2,3$ and the three coordinate systems are obtained in this manner from the angle derivative formulas in the Appendix. Finally, note that as Eq. (43) is independent of $\rho_{L}$ and $P$, the complete integral over all parameters can be expressed as in Eq. (30).

\subsection{Spherical Coordinate System}

The concern that must be addressed in the choice of spherical coordinate system is to avoid adding artificial singularities to the actual ones present in the Green's function. The polar axis is a singular point of the transformation, $\varphi=0$ or $\varphi=\pi$ and any value of $\zeta$ define the same point, and moreover the functions $g_{l, \zeta}$ and $g_{l, \varphi}$ contain denominators which clearly must be kept positive. For the $Z$ coordinate system the denominators contain $a_{1}^{2}+a_{2}^{2}$ and $\beta_{1}^{2}+\beta_{2}^{2}$ (for $X$ and $Y$ systems the subscripts permute appropriately), and thus the $Z$ system cannot be used if either of these quantities can approach zero.

For the integration of the singular term, the Green's function is evaluated for the direction vector $\mathbf{a}$, which is a tangent vector. Thus, the simplest approach is to 
choose the polar axis as the Cartesian vector which lies nearest to the normal vector $\mathbf{N}$. This guarantees that the polar angle $\varphi$ is never close to the troublesome points 0 and $\pi$, and also ensures that the formulas for angle derivatives (evaluated at $\epsilon=0)$ are well behaved.

For the numerical integration of the non-singular term, the Green's function derivatives are also evaluated for the direction vector defined by $\boldsymbol{\beta}(q)$, Eq. (29), a linear combination of $\mathbf{a}$ and the normal $\mathbf{N}$. In this case, the coordinate axis closest to the cross product of these two vectors is chosen for the polar axis. With this choice, the denominators in $g_{l, \zeta}$ and $g_{l, \varphi}$ will not come close to zero. As a depends upon $\theta$, the spherical coordinate system will vary with $\theta$, but this presents no problems for the numerical quadrature.

\subsection{Second integration}

The first analytic integration suffices for the most important task, displaying (and removing) the divergent $\log (\epsilon)$ term, Eq. (22) and Eq. (37). However, it is beneficial to carry out a second exact integration, for two reasons. First, note that $\rho_{L} \approx 0$ for $\xi \approx 0$, and thus Eq. (22) and Eq. (37) contain a logarithmic singularity. This is an integrable singularity for which suitable numerical methods can be employed, but it is simpler and more accurate if an analytic method is used. Second, for the analysis of the hypersingular kernel, a second analytic integration is essential [12], and thus it is a good idea to check that the procedures are successful in this simpler setting.

The $\theta$ dependence of the integrand on $\theta$ in Eq. (22) is harmless; as just discussed, the possible problem is at $\xi=0$. It is therefore necessary to interchange the order of integration, and as in [12], this is accomplished by introducing the variable $t$, $-1 \leq t \leq 1$, via

$$
\theta=-\frac{\pi}{2}+\tan ^{-1}\left(\frac{t-\eta}{\xi}\right) \quad \frac{d \theta}{d t}=\frac{\xi}{\xi^{2}+(t-\eta)^{2}},
$$

which also results in $\rho_{L}=\left(\xi^{2}+(t-\eta)^{2}\right)^{1 / 2}$. As indicated in Figure 2, $t$ is the 'end-point' $(t, 0)$ of $\rho$ on the $\xi^{*}$-axis. The singularity at $\rho_{L}=0$ is now at $t=\eta$, $\xi=0$, and this again suggests polar coordinates $\{\Lambda, \Psi\}$ to replace $\{t, \xi\}$,

$$
\begin{aligned}
t & =\Lambda \cos (\Psi)+\eta \\
\xi & =\Lambda \sin (\Psi),
\end{aligned}
$$

and integrating $\Lambda$. Note that with this transformation $\rho_{L}$ becomes simply $\Lambda$. It is important to note that with the two changes of variables, $\theta \rightarrow t$ and $\{t, \xi\} \rightarrow$ $\{\Lambda, \Psi\}, \cos (\theta)$ becomes $\cos (\Psi)$ and $\sin (\theta)$ becomes $-\sin (\Psi)$. Thus, $a(\theta)$, Eq. (17), becomes $a(\Psi)$ and is a constant as far as the $\Lambda$ integration is concerned. Moreover, 
$\tilde{\mathcal{U}}\left(P_{0}, Q\right)=\tilde{\mathcal{U}}(\mathbf{a}(\theta))$ becomes $\tilde{\mathcal{U}}(\mathbf{a}(\Psi))$, also independent of $\Lambda$. As shown in Fig. 5 , the $\{t, \xi\}$ domain is a rectangle, and integrating over $\{\Lambda, \Psi\}$ will necessitate a decomposition into three subdomains $0 \leq \Psi \leq \Psi_{1}, \Psi_{1} \leq \Psi \leq \pi-\Psi_{2}$, and $\pi-\Psi_{2} \leq \Psi \leq \pi$, where

$$
\begin{aligned}
& \Psi_{1}=\tan ^{-1}\left(\frac{\sqrt{3}(1-|\eta|)}{1-\eta}\right) \\
& \Psi_{2}=\tan ^{-1}\left(\frac{\sqrt{3}(1-|\eta|)}{1+\eta}\right)
\end{aligned}
$$

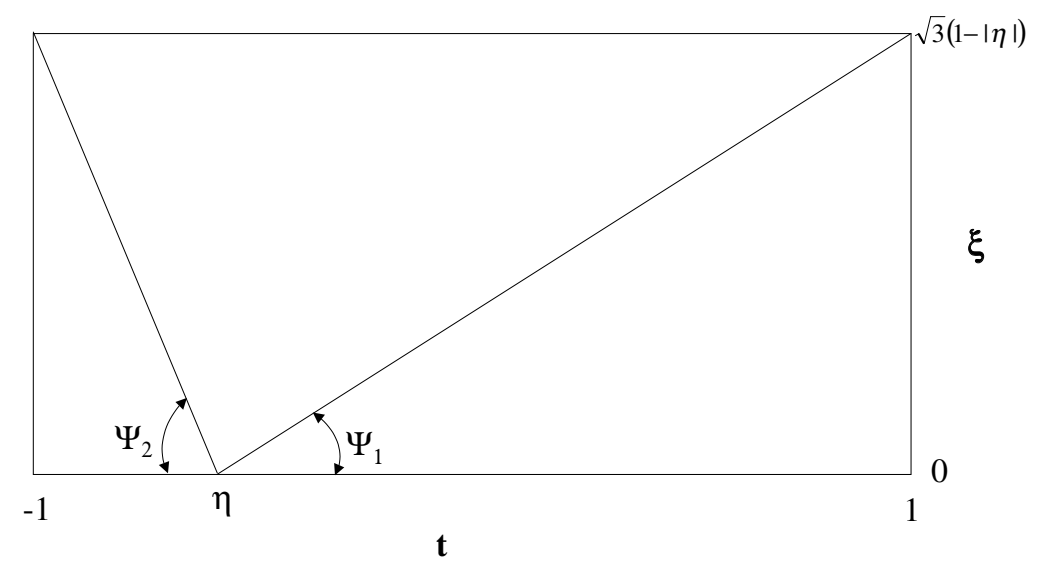

Figure 5: Geometry of the second polar coordinate transformation, $\{t, \xi\} \rightarrow$ $\{\Lambda, \Psi\}$, for the coincident integration.

With this final coordinate transformation, the $P$ shape functions are linear in $\Lambda$, as are the coefficents $c_{j, m}$ from the $Q$ shape functions. The product is quadratic, and the integrals to be evaluated are therefore of the form

$$
\frac{J_{P}^{2}}{8 \pi^{2}} \int_{-1}^{1} \mathrm{~d} \eta \int \frac{a_{l}}{a^{3}} \sin (\Psi) \tilde{\mathcal{U}}(\mathbf{a}) \mathrm{d} \Psi \int_{0} \psi_{k}(\eta, \Lambda, \Psi) \log (2 a \Lambda) \mathrm{d} \Lambda .
$$

The missing limits for the $\Lambda$ and $\Psi$ integrals depend upon the particular subtriangle in Figure 5 being considered. It is now a simple matter to carry out the $\Lambda$ 
integration, and the remaining two dimensional integral over $\{\eta, \Psi\}$ is well behaved and amenable to numerical quadrature.

\section{Edge Adjacent Integration}

For the $\mathcal{U}$ and $\mathcal{T}$ kernels, the singular adjacent edge and vertex integrals are finite with $P \in \Sigma(\epsilon=0)$, and thus a limit process is not required. It is therefore possible to treat these integrals numerically. However, as with the second analytic integration for the coincident integral, it is worthwhile to demonstrate that the methods in [12] apply to anisotropic analysis. Analytic integration is effective in treating the (integrable) singularity and, as noted above, we wish to lay the groundwork for analyzing the more difficult hypersingular kernel. The $\mathcal{T}$ kernel edge-adjacent integral will be briefly discussed below, the simpler $\mathcal{U}$ kernel and the vertex-adjacent integral can be handled in a similar manner.

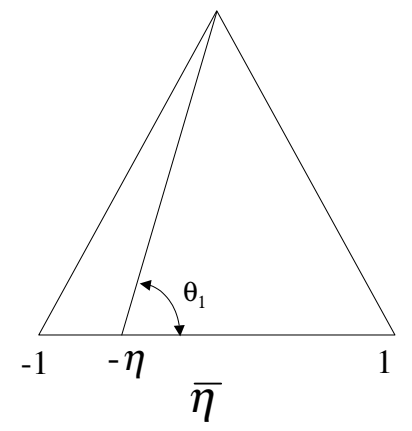

(a)

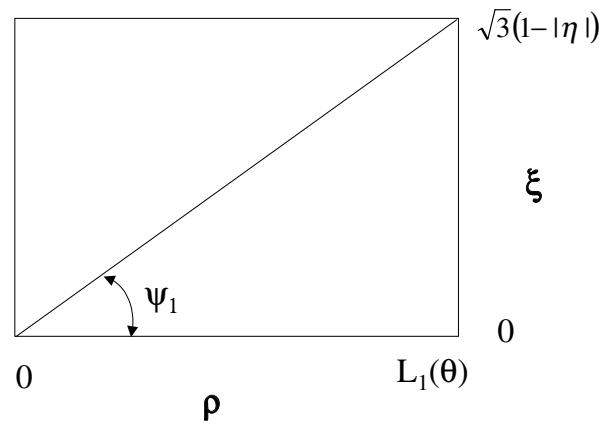

(b)

Figure 6: Polar coordinate transformations employed for the edge-adjacent integration: (a) first transformation in the $Q$ element, $\left\{\eta^{*}, \xi^{*}\right\} \rightarrow\{\rho, \theta\}$; (b) second transformation $\{\rho, \xi\} \rightarrow\{\Lambda, \Psi\}$.

Orient the elements so that the shared edge is defined by $\xi=0$ in $E_{P}$, and $\xi^{*}=0$ for $E_{Q}$, and the singularity occurs when $\eta=-\eta^{*}$. Corresponding to Eq. (14) we 
have

$$
\begin{aligned}
& \frac{J_{P} J_{Q}}{8 \pi^{2}} \int_{-1}^{1} \int_{0}^{e(\eta)} \psi_{k}(\eta, \xi) \mathrm{d} \eta \mathrm{d} \xi \times \\
& \quad \int_{-1}^{1} \int_{0}^{e\left(\eta^{*}\right)} \psi_{j}\left(\eta^{*}, \xi^{*}\right)\left(-\frac{R_{l}}{r^{3}} \tilde{\mathcal{U}}(P, Q)+\frac{1}{r} \frac{\partial}{\partial q_{l}} \tilde{\mathcal{U}}(P, Q)\right) \mathrm{d} \eta^{*} \mathrm{~d} \xi^{*},
\end{aligned}
$$

As illustrated in Figure 6, the first step is to employ polar coordinates for the $Q$ integration,

$$
\begin{aligned}
\eta^{*} & =\rho \cos (\theta)-\eta \\
\xi^{*} & =\rho \sin (\theta) .
\end{aligned}
$$

Denoting the point $P$ by $P(\eta, \xi)$, the center of the polar coordinates is $P_{\eta}=$ $P(\eta, 0)$, the projection of $P$ onto the singular edge. The $\theta$ integration must be split into two parts, $0 \leq \theta \leq \Theta_{1}(\eta)$ and $\Theta_{1}(\eta) \leq \theta \leq \pi$, but the key point is that

$$
\Theta_{1}(\eta)=\frac{\pi}{2}-\tan ^{-1}\left(\frac{\eta}{\sqrt{3}}\right)
$$

depends only on $\eta$. It is therefore possible to execute the $\xi$ integral before the $\theta$ integral, and to introduce the second transformation

$$
\begin{aligned}
& \rho=\Lambda \cos (\Psi) \\
& \xi=\Lambda \sin (\Psi) .
\end{aligned}
$$

Note that $\Lambda=0$ incorporates all three of the conditions for singularity stated above, and that the distance takes the form $r^{2}=b^{2} \Lambda^{2}$; thus, $\tilde{\mathcal{U}}(P, Q)$ is independent of $\Lambda$. Moreover, the combined jacobian for the two transformations is $\cos (\Psi) \Lambda^{2}$, and consequently there is obviously no longer any trace of the singularity. Examining just the $\tilde{\mathcal{U}}$ term from Eq. (51), the derivative term being handled similarly, the $\Lambda$ integral takes the form

$$
-\frac{a_{l}}{b^{3}} \tilde{\mathcal{U}}(P, Q) \int_{0}^{\Lambda_{L}} \psi_{k}(\Lambda, \Psi) \psi_{j}(\Lambda, \Psi) \mathrm{d} \Lambda .
$$

This integral can be computed analytically or numerically. Note that for the hypersingular integral, a limit analysis will be necessary (a $\log (\epsilon)$ singularity is expected), and thus a splitting of integral as in the previous section will be required.

The analysis of the vertex adjacent singular integral follows as above, with appropriate modification to the definition of the polar coordinate transformations that define $\Lambda[12]$.

\subsection{Test Calculation}

While the above analysis stands on its own, it is useful to verify that there are no problems in implementing the above procedures. For the displacement bound- 
ary integral equation, the adjacent edge and vertex integrals, as seen above, are relatively tame. Thus, the main purpose of this exercise is to establish that the coincident integration of the traction kernel is being handled correctly.

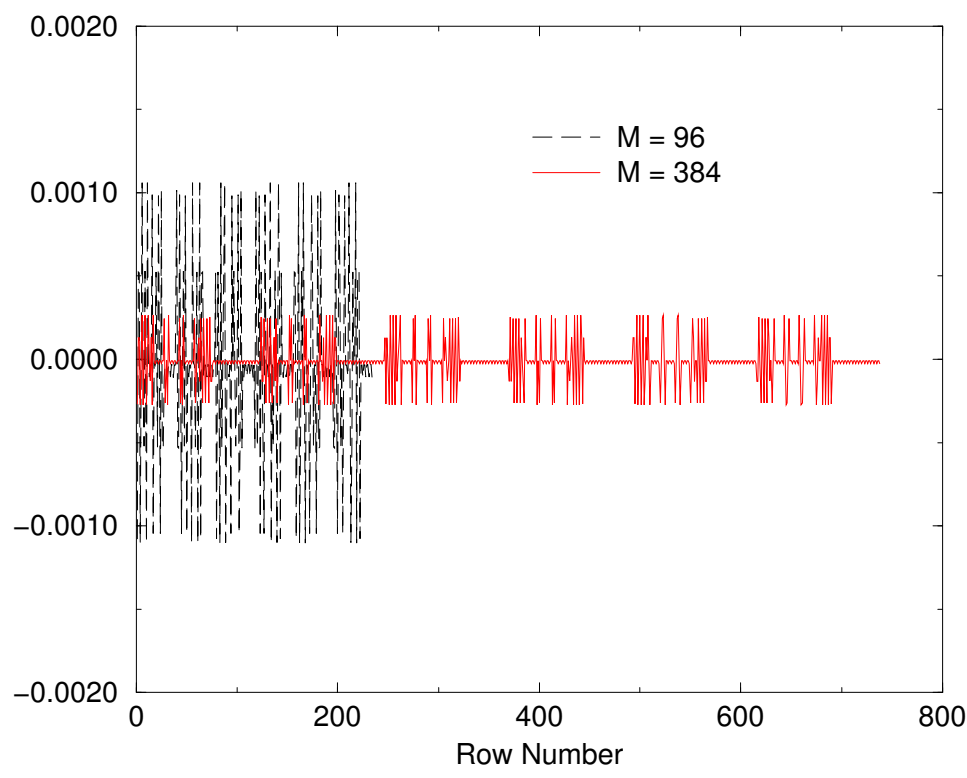

Figure 7: Row sums for the $\mathcal{T}$ matrix for the unit cube problem. $M$ is the number of elements.

A simple test calculation has been performed. The domain is the unit cube, the material is silicon, and the boundary conditions are $\mathbf{u}=0$ on $x=0, \boldsymbol{\tau}=(1,0,0)$ on $x=1$, and $\boldsymbol{\tau}=0$ elsewhere. Two uniform discretizations were employed, one with $M=96$ elements, and one with $M=284$. The code produced reasonably accurate approximate solutions, which improved with the refined grid, as desired. However, a direct way to examine the correctness of the traction kernel integration is to compute the row sums of the matrix representing this integral. In an exact calculation these numbers should be zero, a rigid body motion producing no stress. Figure 7 displays the row sum results for the two meshes, and as desired, substantial improvement is seen for the more refined calculation. 


\section{$5 \quad$ Higher Order Interpolation}

In at least one important application, fracture analysis, it is important to go beyond a linear approximation. The very effective quarter-point crack front element $[3,16,28]$ requires the ability to work with a quadratic interpolation. For Green's functions known in 'algebraic' form, employing the direct limit process for an interpolation beyond linear is more or less straightforward. The primary problem is that the distance $r^{2}$ is no longer a quadratic polynomial in the parametric variables, and thus analytic integration with this denominator is not possible. As described in [11], the basic procedure is to split the kernel function into two components. The first part mimics the form obtained from a linear element: it contains all of the singularity and can be integrated analytically. The remainder is innocuous and can be integrated numerically. The analytical piece is obtained by replacing the troublesome $r^{-m / 2}$ denominators with $\hat{r}^{-m / 2}$, where $\hat{r}^{2}$ takes the linear form, e.g., a simple quadratic.

Implementing this program for the anisotropic Green's function not surprisingly requires an additional step to cope with the presence of $\tilde{\mathcal{U}}$. In this section we briefly describe this procedure, the discussion limited to the coincident integral for the $\mathcal{T}$ kernel, and only the constant term from the $Q$ shape function. The adjacent edge/vertex integrals, and the $\mathcal{U}$ kernel, can be handled in a similar fashion. In addition to complicating the form of the distance function, the higher order interpolation adds terms to the polynomial shape functions multiplying the Green's function. However, the integrals involving these extra terms are finite at $\epsilon=0$, and do not present any difficulties.

Once again, the two terms in the $\mathcal{T}$ kernel will be discussed separately. From Eq. (20), the $\rho$ integral for the first term is

$$
\lim _{\epsilon \rightarrow 0} \int_{0}^{\rho_{L}} \rho \frac{R_{l}}{r^{3}} \tilde{\mathcal{U}}\left(P_{\epsilon}, Q\right) \mathrm{d} \rho,
$$

where now $Q=P_{0}+\rho \mathbf{a}+\rho^{2} \mathbf{b}, \mathbf{b}$ containing all the higher order terms $(i . e ., \mathbf{b}$ can depend upon $\rho$ ). The linear part $P_{0}+\rho \mathbf{a}$ is denoted by $\hat{Q}$, and thus $\hat{r}^{2}=\left\|\hat{Q}-P_{\epsilon}\right\|^{2}$ is, as for the linear element, the quadratic $a^{2} \rho^{2}+\epsilon^{2}$. Rewrite the integrand in Eq. (56) as

$$
\begin{aligned}
& \rho \frac{R_{l}}{r^{3}} \tilde{\mathcal{U}}\left(P_{\epsilon}, Q\right)=\rho \frac{R_{l}}{\hat{r}^{3}} \tilde{\mathcal{U}}\left(P_{\epsilon}, \hat{Q}\right)+ \\
& \quad \rho\left[\frac{R_{l}}{r^{3}}-\frac{R_{l}}{\hat{r}^{3}}\right] \tilde{\mathcal{U}}\left(P_{\epsilon}, Q\right)+\rho \frac{R_{l}}{\hat{r}^{3}}\left[\tilde{\mathcal{U}}\left(P_{\epsilon}, Q\right)-\tilde{\mathcal{U}}\left(P_{\epsilon}, \hat{Q}\right)\right] .
\end{aligned}
$$

The first term on the right hand side has the same form as the linear element expression, except that $R_{l}$ will contain higher order terms in $\rho$. It can therefore 
be dealt with as in Section 3. In the second term, $P_{\epsilon}$ can be replaced by $P_{0}$,

$$
\rho\left[\frac{R_{l}}{r^{3}}-\frac{R_{l}}{\hat{r}^{3}}\right] \tilde{\mathcal{U}}\left(P_{0}, Q\right),
$$

and integrated numerically. This follows by observing that $\rho R_{l}\left(r^{-3}-\hat{r}^{-3}\right)$ is well behaved at $\epsilon=0$, and that $\tilde{\mathcal{U}}\left(P_{\epsilon}, Q\right)-\tilde{\mathcal{U}}\left(P_{0}, Q\right)$ goes to zero for $\epsilon \rightarrow 0$, except at $\rho=0$ where it has the constant value $\tilde{\mathcal{U}}(\mathbf{N})-\tilde{\mathcal{U}}(\mathbf{a})$. Thus,

$$
\lim _{\epsilon \rightarrow 0} \int_{0}^{\rho_{L}} \rho\left(\frac{R_{l}}{r^{3}}-\frac{R_{l}}{\hat{r}^{3}}\right)\left[\tilde{\mathcal{U}}\left(P_{\epsilon}, Q\right)-\tilde{\mathcal{U}}\left(P_{0}, Q\right)\right] \mathrm{d} \rho=0 .
$$

For the last term in Eq. (57), rewrite the $\tilde{\mathcal{U}}$ factor as the two components

$$
\begin{aligned}
& \tilde{\mathcal{U}}\left(P_{\epsilon}, Q\right)-\tilde{\mathcal{U}}\left(P_{\epsilon}, \hat{Q}\right)=\tilde{\mathcal{U}}\left(P_{0}, Q\right)-\tilde{\mathcal{U}}\left(P_{0}, \hat{Q}\right)+ \\
& \quad\left(\left[\tilde{\mathcal{U}}\left(P_{\epsilon}, Q\right)-\tilde{\mathcal{U}}\left(P_{0}, Q\right)\right]-\left[\tilde{\mathcal{U}}\left(P_{\epsilon}, \hat{Q}\right)-\tilde{\mathcal{U}}\left(P_{0}, \hat{Q}\right)\right]\right) .
\end{aligned}
$$

Note that $\tilde{\mathcal{U}}\left(P_{0}, Q\right)-\tilde{\mathcal{U}}\left(P_{0}, \hat{Q}\right)=0$ at $\rho=0$. It is therefore possible to shift a factor of $\rho$ in the integrand and then set $\epsilon=0$, i.e.,

$$
\begin{aligned}
\lim _{\epsilon \rightarrow 0} & \int_{0}^{\rho_{L}} \rho \frac{R_{l}}{\hat{r}^{3}}\left[\tilde{\mathcal{U}}\left(P_{0}, Q\right)-\tilde{\mathcal{U}}\left(P_{0}, \hat{Q}\right)\right] \mathrm{d} \rho \\
& =\left.\int_{0}^{\rho_{L}} \frac{\rho^{2} R_{l}}{\hat{r}^{3}}\right|_{\epsilon=0}\left[\frac{\tilde{\mathcal{U}}\left(P_{0}, Q\right)-\tilde{\mathcal{U}}\left(P_{0}, \hat{Q}\right)}{\rho}\right] \mathrm{d} \rho \\
& =\int_{0}^{\rho_{L}} \frac{a_{l}+b_{l} \rho}{a^{3}}\left[\frac{\tilde{\mathcal{U}}\left(P_{0}, Q\right)-\tilde{\mathcal{U}}\left(P_{0}, \hat{Q}\right)}{\rho}\right] \mathrm{d} \rho .
\end{aligned}
$$

This last integral can be evaluated numerically. Finally, the integral of the second component in Eq. (60) is zero: this function is not only zero at $\rho=0$, thus contributing the needed factor of $\rho$ to remove the singularity, but it also vanishes for $\epsilon \rightarrow 0$.

To summarize, for a curved interpolation,

$$
\begin{aligned}
& \lim _{\epsilon \rightarrow 0} \int_{0}^{\rho_{L}} \rho \frac{R_{l}}{r^{3}} \tilde{\mathcal{U}}\left(P_{\epsilon}, Q\right) \mathrm{d} \rho=\lim _{\epsilon \rightarrow 0} \int_{0}^{\rho_{L}} \rho \frac{R_{l}}{\hat{r}^{3}} \tilde{\mathcal{U}}\left(P_{\epsilon}, \hat{Q}\right) \mathrm{d} \rho+ \\
& \quad \int_{0}^{\rho_{L}} \rho\left(\frac{R_{l}}{r^{3}}-\frac{R_{l}}{\hat{r}^{3}}\right) \tilde{\mathcal{U}}\left(P_{0}, Q\right) \mathrm{d} \rho+\int_{0}^{\rho_{L}} \frac{a_{l}+b_{l} \rho}{a^{3}}\left[\frac{\tilde{\mathcal{U}}\left(P_{0}, Q\right)-\tilde{\mathcal{U}}\left(P_{0}, \hat{Q}\right)}{\rho}\right] \mathrm{d} \rho .
\end{aligned}
$$

The last two integrals are safely evaluated numerically, while as noted above, the first is handled as in the linear element analysis. Note that analytic integration is only invoked for this 'linear element' contribution. The second analytic evaluation will therefore only proceed with this term, executed as described in section 3.4. 
The integral for the other half of $\mathcal{T}$,

$$
\lim _{\epsilon \rightarrow 0} \int_{0}^{\rho_{L}} \frac{\rho}{r} \frac{\partial}{\partial q_{l}} \tilde{\mathcal{U}}\left(P_{\epsilon}, Q\right) \mathrm{d} \rho
$$

can be handled in the same manner. The reworking of the $\tilde{\mathcal{U}}$ factor in fact follows precisely as above; the only aspect that requires a brief comment is the treatment of the angle derivative functions. For discussion purposes, we once again consider $l=1$, the $Z$ spherical coordinate system, and the $\zeta$ derivative. The algebraic coefficient in this case (replacing $\rho R_{l} / r^{3}$ in the above discussion) is

$$
\frac{\rho}{r} \frac{\partial \zeta}{\partial q_{1}}=-\frac{\rho}{r} \frac{q_{2}-p_{2}}{\left(q_{1}-p_{1}\right)^{2}+\left(q_{2}-p_{2}\right)^{2}} \equiv-\left(q_{2}-p_{2}\right) \frac{\rho}{r} \delta
$$

The appropriate 'linear element' approximation of this function is

$$
\frac{\rho}{r} \frac{\partial \zeta}{\partial q_{1}} \approx-\frac{\rho}{\hat{r}} \frac{q_{2}-p_{2}}{\left(\hat{q}_{1}-p_{1}\right)^{2}+\left(\hat{q}_{2}-p_{2}\right)^{2}} \equiv-\left(q_{2}-p_{2}\right) \frac{\rho}{\hat{r}} \hat{\delta}
$$

it being permissible to keep the higher order terms in $q_{2}$ in the numerator. This approximate term is sufficiently simple that it can be integrated analytically. To complete the discussion, it is necessary to show that the remainder term can be integrated numerically, i.e., that it is well behaved in the limit. This follows immediately from

$$
\rho\left(q_{2}-p_{2}\right)\left[\frac{\delta}{r}-\frac{\hat{\delta}}{\hat{r}}\right]=\rho\left(q_{2}-p_{2}\right)\left[\left(\frac{1}{r}-\frac{1}{\hat{r}}\right) \delta+(\delta-\hat{\delta}) \frac{1}{\hat{r}}\right] .
$$

\section{Conclusions}

Despite the fact that the Green's function for three dimensional anisotropic elasticity is not known in closed form, singular integration procedures based upon a boundary limit and analytic integration can be carried out. In the limit process, the angular variation of the Green's function, $\tilde{\mathcal{U}}$, is handled by splitting it into two terms. In the first, the $\tilde{\mathcal{U}}$ factor is independent of the integration variables, and thus analytic integration of this singular term can proceed. The remaining term is non-singular and can be expressed in a form suitable for numerical evaluation.

The methods were shown to be successful for the displacement $\mathcal{U}(P, Q)$ and traction $\mathcal{T}(P, Q)$ kernels in the boundary integral equation for surface displacement. However, the goal is to address the hypersingular equation for surface traction using the similar techniques. In this regard, procedures that will be necessary for the traction equation, namely two analytic integrations for the coincident integral and two polar coordinate transformations for the adjacent edge integral, were implemented for the displacement equation. 
Based upon these results, it is expected that the direct limit method can be successfully applied to the traction equation, providing an alternative to the Stokes' Theorem approach recently developed by Mear [23]. In this analysis however, it will not be as easy, as in Eq. (23) and Eq. (37), to materialize the potentially divergent terms, and to establish that they disappear. As in the analysis of the Laplace hypersingular equation [12], the coincident integration must produce $1 / \epsilon$ and $\log (\epsilon)$ divergent terms: the first must self-cancel, while the second must cancel with a corresponding term from the edge adjacent integral. However, due to the complexity of the hypersingular kernel function, establishing these results will not be trivial.

\section{Acknowledgment}

This research was supported by the Applied Mathematical Sciences Research Program of the Office of Mathematical, Information, and Computational Sciences, U.S. Department of Energy, under contract DE-AC05-00OR22725 with UTBattelle, LLC. A. Griffith and L. Johnson acknowledge support from undergraduate research programs, ERULF and HERE, respectively, at Oak Ridge National Laboratory. The authors would like to thank Profs. M. Bonnet and P. A. Martin for pointing out references [4] and [36], respectively.

The submitted manuscript has been authored by a contractor of the U. S. Government under contract DE-AC05-00OR22725. Accordingly the U. S. Government retains a non-exclusive, royalty free license to publish or reproduce the published form of this contribution, or allow others to do so, for U. S. Government purposes.

\section{References}

[1] A. Aimi And M. Diligenti, Hypersingular kernel integration in 3D Galerkin boundary element method, J. Comput. Appl. Math., 138 (2002), pp. 51-72.

[2] D. M. BARNetT, The precise evaluation of derivatives of the anisotropic elastic Green's functions, Phys. Stat. Sol. (b), 49 (1972), pp. 741-748.

[3] R. S. BARsoum, On the use of isoparametric finite elements in linear fracture mechanics, Int. J. Numer. Meth. Engrg., 10 (1976), pp. 25-37.

[4] E. Becache, J.-C. Nedelec, and N. Nishimura, Regularization in 3D for anisotropic elastodynamic crack and obstacle problems, J. Elasticity, 31 (1993), pp. 25-46.

[5] M. Bonnet, Boundary Integral Equation Methods for Solids and Fluids, Wiley and Sons, England, 1995. 
[6] A. Carini and A. Salvadori, Analytical integrations 3D BEM, Comp. Mech. in press.

[7] A. Deb, J. D. P. Henry, And R. B. Wilson, Alternate BEM formulations for 2- and 3-d thermoelasticity, Int. J. Solids Structures, 27 (1991), pp. 17211738.

[8] V. J. Ervin and E. P. Stephan, A boundary element Galerkin method for a hypersingular integral equation on open surfaces, Math. Meth. Appl. Sci., 13 (1990), pp. 281-289.

[9] A. Frangi, Regularization of boundary element formulations by the derivative transfer method, in Singular Integrals in the Boundary Element Method, V. Sladek and J. Sladek, eds., Advances in Boundary Elements, Computational Mechanics Publishers, 1998, ch. 4, pp. 125-164.

[10] A. Frangi, G. Novati, R. Springhetti, and M. Rovizzi, $3 d$ fracture analysis by the symmetric Galerkin BEM, Computational Mechanics, 28 (2002), pp. 220-232.

[11] L. J. Gray, Evaluation of singular and hypersingular Galerkin boundary integrals: direct limits and symbolic computation, in Singular Integrals in the Boundary Element Method, V. Sladek and J. Sladek, eds., Advances in Boundary Elements, Computational Mechanics Publishers, 1998, ch. 2, pp. 33-84.

[12] L. J. Gray, J. Glaeser, And T. Kaplan, Direct evaluation of hypersingular Galerkin surface integrals, SIAM J. Sci. Comput., (2004). in press.

[13] L. J. Gray and T. Kaplan, 3D Galerkin integration without Stokes' Theorem, Engineering Analysis with Boundary Elements, 25 (2001), pp. 289-295.

[14] M. Guiggiani, The evaluation of cauchy principal value integrals in the boundary element method - a review, Mathematical and Computer Modelling, 15 (1991), pp. 175-184.

[15] S. A. Gundersen And J. Lothe, A new method for numerical claculations in anisotropic elasticity problems, Phys. Stat. Sol. (b), 143 (1987), pp. 73-85.

[16] R. D. Henshell and K. G. Shaw, Crack tip finite elements are unnecessary, Int. J. Numer. Meth. Engrg., 9 (1975), pp. 495-507.

[17] G. Krishnasamy, F. J. Rizzo, and T. J. Rudolphi, Hypersingular boundary integral equations: Their occurrence, interpretation, regularization and computation, in Developments in Boundary Element Methods - Advanced Dynamic Analysis, P. K. Banerjee and S. Kobayashi, eds., vol. 7, Elsevier Applied Science Publishers, 1991, ch. 7. 
[18] S. Li, M. E. Mear, And L. XiaO, Symmetric weak form integral equation method for three-dimensional fracture analysis, Comp. Meth. Appl. Mech. Engng., 151 (1998), pp. 435-459.

[19] I. M. Lifschitz And L. N. Rozentsweig, Zh. Eksperim. Teor. Fiz., 17 (1947), p. 783.

[20] E. D. Lutz And L. J. Gray, Exact evaluation of singular boundary integrals without CPV, Comm. Num. Meth. Engrg., 9 (1993), pp. 909-915.

[21] G. Maier, M. Diligenti, And A. Carini, A variational approach to boundary element elastodynamic analysis and extension to multidomain problems, Comp. Meth. Appl. Engng., 92 (1991), pp. 193-213.

[22] K. Malén, Phys. Stat. Sol. (b), 49 (1972), pp. 661-672.

[23] M. MEAR, personal communication, (2003).

[24] M.G.DufFy, Quadrature over a pyramid or cube of integrands with a singularity at a vertex, SIAM J Numer. Analy., 19 (1982), pp. 1260-1262.

[25] G. Nakamura and K. Tanuma, A formula for the fundamental solution of anisotropic elasticity, Q. J. Mech. Appl. Math, 50 (1997), pp. 179-194.

[26] F. W. J. Olver, Bessel functions of integer order, in Handbook of mathematical functions, M. Abromowitz and I. A. Steegun, eds., National Bureau of Standards, Washington, D.C., 1972, ch. 9, pp. 355-434.

[27] E. PAn AND F. G. YuAn, Three-dimensional Green's functions in anisotropic bimaterials, I. J. Solids Struct., 37 (2000), pp. 5329-5351.

[28] A.-V. Phan, L. J. Gray, G. H. Paulino, and T. Kaplan, Improved quarter-point crack tip element, Engineering Fracture Mechanics. in press.

[29] M. A. Sales And L. J. Gray, Evaluation of the anisotropic Green's function and its derivatives, Computers and Structures, 69 (1998), pp. 247-254.

[30] A. SAlVADORI, Quasi brittle fracture mechanics by cohesive crack models and symmetric Galerkin boundary element method, $\mathrm{PhD}$ thesis, Politecnico Milano, Milan, Italy, 1999.

[31] — Analytical integrations of hypersingular kernel in 3D BEM problems, Comp. Meth. in Appl. Mech. Engng., 190 (2001), pp. 3957-3975.

[32] N. A. SchClar, Anisotropic Analysis using Boundary Elements, Computational Mechanics Publications, Southampton and Boston, 1994.

[33] C. Schwab And W. L. Wendland, On numerical cubatures of singular integrals in boundary element methods, Numer. Math., 62 (1992), pp. 343369. 
[34] S. Sirtori, G. Maier, G. Novati, and S. Miccoli, A Galerkin symmetric boundary element method in elasticity: formulation and implementation, Int. J. Numer. Meth. Engrg., 35 (1992), pp. 255-282.

[35] V. Sladek and J. Sladek, eds., Singular integrals in boundary element methods, Advances in Boundary Elements, Computational Mechanics Publications, 1998.

[36] J. L. Synge, The Hypercircle in Mathematical Physics, Cambridge University Press, London, 1957.

[37] M. Tanaka, V. Sladek, and J. Sladek, Regularization techniques applied to boundary element methods, Applied Mechanics Reviews, 47 (1994), pp. 457499.

[38] T. C. T. Ting and V.-G. LeE, The three-dimensional elastostatic Green's function for general anisotropic linear elastic solids, Q. J. Mech. Appl. Math, 50 (1997), pp. 407-426.

[39] S. M. Vogel And F. J. Rizzo, An integral equation formulation of threedimensional anisotropic elastostatic boundary value problems, J. Elast., 3 (1973), pp. 203-216.

[40] C.-Y. WANG, Elastic fields produced by a point source in solids of general anisotropy, J. Engng. Math., 32 (1997), pp. 41-52.

[41] R. B. Wilson And T. A. CRuse, Efficient implementation of anisotropic three dimensional boundary-integral equation stress analysis, Int. J. Numer. Meth. Engrg., 12 (1978), pp. 1383-1397.

\section{Appendix}

\subsection{Angle Derivatives}

For the three coordinate systems, with $\{Z, Y, X\}$ as polar direction, the spherical angles $\{\zeta, \varphi\}, 0<\zeta<2 \pi, 0<\varphi<\pi$ are defined respectively via

$$
\begin{aligned}
\left(\frac{q_{1}-p_{1}}{r}, \frac{q_{2}-p_{2}}{r}, \frac{q_{3}-p_{3}}{r}\right) & =(\cos (\zeta) \sin (\varphi), \sin (\zeta) \sin (\varphi), \cos (\varphi)) \\
& =(\sin (\zeta) \sin (\varphi), \cos (\varphi), \cos (\zeta) \sin (\varphi)) \\
& =(\cos (\varphi), \cos (\zeta) \sin (\varphi), \sin (\zeta) \sin (\varphi))
\end{aligned}
$$


where the distance is

$$
r=\left(\left(q_{1}-p_{1}\right)^{2}+\left(q_{2}-p_{2}\right)^{2}+\left(q_{3}-p_{3}\right)^{2}\right)^{1 / 2} .
$$

The angle derivatives $\frac{\partial \zeta}{\partial q_{l}}$ and $\frac{\partial \varphi}{\partial q_{l}}$ are obtained by straightforward differentiation. For the $Z$ coordinate system these are

$$
\begin{array}{ll}
\frac{\partial \zeta}{\partial q_{1}}=-\frac{q_{2}-p_{2}}{\left(q_{1}-p_{1}\right)^{2}+\left(q_{2}-p_{2}\right)^{2}} & \frac{\partial \varphi}{\partial q_{1}}=\frac{\left(q_{1}-p_{1}\right)\left(q_{3}-p_{3}\right)}{r^{2}\left[\left(q_{1}-p_{1}\right)^{2}+\left(q_{2}-p_{2}\right)^{2}\right]^{1 / 2}} \\
\frac{\partial \zeta}{\partial q_{2}}=\frac{q_{1}-p_{1}}{\left(q_{1}-p_{1}\right)^{2}+\left(q_{2}-p_{2}\right)^{2}} & \frac{\partial \varphi}{\partial q_{2}}=\frac{\left(q_{2}-p_{2}\right)\left(q_{3}-p_{3}\right)}{r^{2}\left[\left(q_{1}-p_{1}\right)^{2}+\left(q_{2}-p_{2}\right)^{2}\right]^{1 / 2}} \\
\frac{\partial \zeta}{\partial q_{3}}=0 & \frac{\partial \varphi}{\partial q_{3}}=-\frac{\left(q_{1}-p_{1}\right)^{2}+\left(q_{2}-p_{2}\right)^{2}}{r^{2}\left[\left(q_{1}-p_{1}\right)^{2}+\left(q_{2}-p_{2}\right)^{2}\right]^{1 / 2}}
\end{array}
$$

The formulas for the other two coordinate systems are obtained by suitably permuting the indices,

$$
\begin{aligned}
\frac{\partial \zeta^{Y}}{\partial q_{\sigma(l)}}\left(r_{j}\right) & =\frac{\partial \zeta^{Z}}{\partial q_{l}}\left(r_{\sigma(j)}\right) \\
\frac{\partial \zeta^{X}}{\partial q_{\sigma^{2}(l)}}\left(r_{j}\right) & =\frac{\partial \zeta^{Z}}{\partial q_{l}}\left(r_{\sigma^{2}(j)}\right)
\end{aligned}
$$

and similarly for $\varphi$. Here $r_{j}=q_{j}-p_{j}$ and the permutation $\sigma$ is defined by $\sigma(1)=3$, $\sigma(2)=1$, and $\sigma(3)=2$. 